\title{
Aspectos psicosociales de la enfermedad celíaca en España: una vida libre de gluten
}

\author{
Psychosocial aspects of celiac disease in \\ Spain: A life free of gluten
}

\author{
Julián Rodríguez ALMAGRO \\ Gonzalo BACIGALUPE² \\ María Carmen Solano RUIZ ${ }^{3}$ \\ José Siles GONZÁLEZ ${ }^{3}$ \\ Antonio Hernández MARTíNEZ ${ }^{4}$
}

\section{R E S U M E N}

\section{Objetivo}

La celiaquía como enfermedad crónica tiene una alta prevalencia en nuestra sociedad. El artículo analiza los aspectos psicosociales de la enfermedad celíaca en los diferentes entornos, valorando el impacto de la implantación de una dieta estricta libre de gluten.

\section{Métodos}

Estudio cualitativo entre los meses de Enero del 2013 a Abril del 2013 en el cual, a través de la teoría fundamentada y el análisis de contenido, se ha profundizado en el análisis de las entrevistas semiestructuradas a personas con enfermedad celíaca en España. Se realizaron llamamientos a traves de las redes sociales y grupos de celíacos hasta llegar a la saturación teórica, que determinó el tamaño final de la muestra de 24 personas.

\section{Resultados}

Educación Sanitaria, Aislamiento social, soledad y desconocimiento social emergieron como categorías centrales en la experiencia de adopción de la dieta libre de gluten a partir del diagnóstico de celiaquía.

\section{Conclusión}

Los profesionales deben promover y apoyar estrategias de apoyo social basadas en una comprensión íntegra de las experiencias de desarraigo y marginación que los pacientes celíacos experimentan en sus relaciones sociales

\footnotetext{
1 Universitario de Ciudad Real, Hospital General, Servicio Urgencias. Calle del Obispo Rafael Torija, s/n., 13005, Ciudad Real, Ciudad Real, Espanã. Dirección de Correspondencia/Correspondence to: JR ALMAGRO. E-mail: <jupij79@gmail.com>.

2 University of Massachusetts Boston, College of Education and Human Development, Department of Counseling and School Psychology. Boston, Massachusetts, EEUU.

3 Universidad de Alicante, Facultad Enfermería, Departamento Enfermería. Alicante, España.

${ }^{4}$ Hospital General Mancha-Centro, Unidad Docente de Matronas. Alcázar de San Juan, Ciudad Real, España.

Artículo elaborado a partir de la disertación de JR ALMAGRO intitulada "Experiencia y calidad de vida de la Celiaquía en España a través de un diseño Mixto", Universidad de Alicante; 2016.
} 
a través de la comida. Se requiere un aumento de la educación sanitaria para entender e integrar el impacto psicosocial del diagnóstico de celiaquía y la dieta libre de gluten.

Palabras clave: Dieta sin gluten. Enfermedad celíaca. Investigación cualitativa. Percepción social.

\section{A B S T R A C T}

\section{Objective}

Celiac disease is a chronic disease which has a high prevalence in our society. The aim of this paper is to explore the process of adapting the celiac in different social environments, assessing the impact of the implementation of a strict gluten-free diet.

\section{Methods}

A qualitative study was carried out in the months of January 2013 to April 2013 through which the grounded theory and content analysis have deepened the analysis of semi-structured interviews with people with celiac disease in Spain. Appeals via social networks and celiac groups were conducted in order to reach theoretical saturation, which determined the final sample size of 24 people.

\section{Results}

Social isolation, loneliness and social ignorance are some of the categories that have emerged when we talk about celiac disease and the gluten-free diet.

\section{Conclusion}

Professionals should promote and support social support strategies based on an integrated understanding of the experiences of displacement and marginalization that celiac patients experience in their social relationships through food. There should be increased health education to understand and integrate the psychosocial impact of the diagnosis of celiac disease and the gluten-free diet.

Keywords: Diet, gluten-free. Celiac disease. Qualitative research. Social perception.

\section{INTRODUCCIÓN}

La Enfermedad Celíaca (EC) es una enfermedad crónica del aparato digestivo que se define como una Intolerancia Permanente al Gluten, que, en individuos predispuestos genéticamente, ocasiona una lesión en la mucosa del intestino delgado'. Se estima que la prevalencia a nivel mundial varía entre 1:100 y 1:300 personas, dependiendo del país ${ }^{2-4}$, y esta prevalencia es significativamente más alta que hace 20 años ${ }^{5,6}$. La relación entre hombres y mujeres es 2:1. La intervención nutricional en un enfermo celíaco implica la instauración de un plan alimentario libre de gluten; si la intolerancia permanente al gluten ha sido confirmada con sus respectivas biopsias, se deberá mantener durante toda la vida ${ }^{7,8}$.

En condiciones normales, el intestino delgado está recubierto de protuberancias microscópicas llamadas vellosidades intestinales, que son las encargadas de la absorción de los alimentos. Cuando se introduce el gluten se produce, en los individuos predispuestos, una atrofia severa de las vellosidades, que conlleva una mala absorción de los nutrientes: proteínas, grasas, hidratos de carbono, sales minerales y vitaminas, con una repercusión clínica muy variable $^{9}$. El gluten es una proteína que se encuentra en los cereales: trigo (gliadina), cebada (hordeina), centeno (secalina), triticale (hibrido de trigo y centeno), y posiblemente avena (avenina). Sin embargo, existen cereales como el arroz, quinua, maíz, mijo, o sorgo, los cuales no contienen gluten, por lo que estos cereales pueden ser consumidos por los enfermos celíacos 9 .

El seguimiento de una dieta libre de gluten requiere la colaboración del celíaco, que necesita tener un conocimiento claro, tanto de la enfermedad como de los alimentos que puede o 
no consumir. Existen estrategias comunes para el mantenimiento de una dieta libre de gluten como es la lectura de los ingredientes en los productos, el etiquetado correcto que indique que el producto es libre de gluten, llevar siempre encima tanto en la escuela como en el trabajo productos libres de gluten, y llevar su propia comida a eventos sociales ${ }^{10}$.

El momento evolutivo de la enfermedad es condicionante de las medidas terapéuticas a aplicar, teniendo en cuenta la situación fisiopatológica, el grado de nutrición y los requerimientos propios de cada edad, además del aumento de un considerable gasto en alimentación ya que de ello dispone la continuidad de una buena salud ${ }^{11}$. Dependiendo de la edad del paciente, lo primero que se hace imprescindible, es la toma de conciencia de lo que acontece, seguido de la aceptación del problema. Esto se hace extensivo a la familia, quien va servir de soporte en esta nueva "tarea de planear un menú libre de gluten y equilibrado" 12 .

Las enfermedades crónicas como la celiaquía hacen que las familias se encuentren con dificultades a la hora de las comidas ${ }^{13,14}$. La preparación de las comidas y el intercambio no solo constituyen un proceso biológico, sino que también conllevan un proceso de relacionarse con la gente y una experiencia sociocultural ${ }^{15}$. La carencia de limitaciones físicas, en el caso de una dieta correcta y de la inexistencia de un deterioro progresivo, sitúan a la EC como una enfermedad crónica privilegiada frente a muchas otras, aunque no podemos subestimar el efecto psicológico que puede tener en los sujetos con enfermedad celíaca. Hay que estimar la limitación real que representa para el sujeto y su familia.

El objetivo principal de esta investigación cualitativa fue analizar los problemas de adaptación del celíaco en los diferentes entornos sociales. Se busca además valorar el impacto de la implantación de una dieta libre de gluten en su vida diaria.

\section{M É T O D O S}

\section{Diseño}

La estrategia metodológica empleada para la consecución de estos objetivos es cualitativa, y se basa en la aplicación de la entrevista individual semiestructurada. A través de la teoría fundamentada y el análisis de contenido se han extraído una serie de categorías, no obstante se ha diseñado un programa metodológico en la investigación que incluye una combinación de métodos para lograr una visión más precisa de la problemática planteada que aborda ${ }^{16}$. La Teoría Fundamentada tiene como objetivo crear categorías teóricas a partir de los datos y analizar las relaciones relevantes que hay entre ellas ${ }^{17}$. Es decir, a través de los procedimientos analíticos, se construye una teoría que está fundamentada en los datos, de ahí su nombre ${ }^{18}$.

Se utilizaron entrevistas en profundidad y los métodos de la Teoría Fundamentada para recoger y analizar los datos ${ }^{19,20}$. La recolección de datos finalizó cuando hubo redundancia de información y las categorías se saturaron teóricamente ${ }^{21}$. Se eligió este método inductivo con el fin de obtener una explicación teórica a partir del análisis de las conceptualizaciones de los participantes sobre la celiaquía. La triangulación metodológica a través del análisis de entrevistas, documentos, y anotaciones de campo permitió verificar coincidencias entre discursos (entrevistas) y acciones (documentos y anotaciones) ${ }^{19}$, no obstante en el análisis de datos solo se hace referencia a las entrevistas, ya que las notas de campo son muy extensas y en su análisis nos indican los mismos resultados que fortalecen los resultados del análisis de las entrevistas. La participación de varios investigadores en el análisis asegura más confiabilidad junto a la inclusión de participantes de diferentes características.

En total se realizaron 24 entrevistas semiestructuradas en toda España, y se recogió una serie de características sociodemográficas 
como edad, sexo, y tiempo diagnóstico de la Enfermedad Celíaca (Tabla 1). Estas entrevistas siguieron un guión semiestructurado (Cuadro 1), tuvieron una duración de entre 60 y 90 minutos y se realizaron durante los meses de Enero a abril de 2013, tiempo en el que los pacientes celíacos expresaron en sus propios términos la experiencia de salir a comer fuera de casa y la consiguiente dieta libre de gluten, transcribiéndola en su totalidad.

Las citas en los resultados utilizan la siguiente codificación: la primera letra "E" corresponde a entrevista, a continuación se coloca un número que va desde el 01 hasta el 24 correspondiente al orden de realización de la entrevista, y por último la letra " $\mathrm{H}$ " correspondiente a hombre o la letra " $M$ " correspondiente a mujer. El muestreo fue de carácter intencional, realizando entrevistas a aquellas personas celíacas adultas que se ponían en contacto con el equipo investigador tras haber visto los llamamientos realizados en las redes sociales. Aunque el tamaño de la muestra teórica, fue insuficiente para garantizar la validez externa en términos de otros modelos de investigación ${ }^{20}$, fue adecuado para saturar todas las categorías incluyendo participantes de diversas características sociodemográficas.

Durante los meses de Mayo a Julio del 2013 se usó metodología cualitativa para el análisis de datos. No obstante haber sido utilizada en multitud de disciplinas y contextos, la investigación cualitativa se refiere siempre al análisis de la realidad social y cultural, que de esta manera nos sitúa en el paradigma fenomenológico, o sea, aquel que pretende comprender los fenómenos sociales desde la propia perspectiva del actor, a diferencia de la perspectiva positivista, que busca los hechos o causas de los fenómenos sociales con independencia de los estados subjetivos de los individuos ${ }^{22}$.

Tabla 1. Características sociodemográficas de las personas celíacas. España, 2013.

\begin{tabular}{|c|c|c|c|c|}
\hline \multirow{2}{*}{ Entrevista } & \multicolumn{2}{|c|}{ Sexo $(n=24)$} & \multirow{2}{*}{ EDAD (Años) } & \multirow{2}{*}{ Tiempo CON EC } \\
\hline & Mujeres(n=18) & Hombres $(n=6)$ & & \\
\hline 1 & Mujer & & 22 & 2 meses \\
\hline 2 & Mujer & & 24 & 2 años \\
\hline 3 & Mujer & & 31 & 3 años e 5 meses \\
\hline 4 & Mujer & & 39 & 2 años \\
\hline 5 & Mujer & & 25 & 6 meses \\
\hline 6 & Mujer & & 36 & 9 meses \\
\hline 7 & Mujer & & 36 & 1 año \\
\hline 8 & & Hombre & 45 & 6 meses \\
\hline 9 & & Hombre & 37 & 6 años \\
\hline 10 & & Hombre & 42 & 24 años \\
\hline 11 & Mujer & & 18 & 6 años \\
\hline 12 & Mujer & & 23 & 2 años \\
\hline 13 & Mujer & & 31 & 3 años \\
\hline 14 & Mujer & & 26 & 3 años \\
\hline 15 & Mujer & & 35 & 1 año \\
\hline 16 & Mujer & & 62 & 28 años \\
\hline 17 & & Hombre & 39 & 3 años \\
\hline 18 & Mujer & & 41 & 2 años \\
\hline 19 & Mujer & & 25 & 2 años \\
\hline 20 & & Hombre & 15 & 12 años \\
\hline 21 & Mujer & & 29 & 11 años \\
\hline 22 & Mujer & & 25 & 2 años \\
\hline 23 & & Hombre & 18 & 2 años \\
\hline 24 & Mujer & & 31 & 3 años \\
\hline
\end{tabular}


Cuadro 1. Guión de preguntas utilizado para modular la entrevista. España (Enero-Abril, 2013).

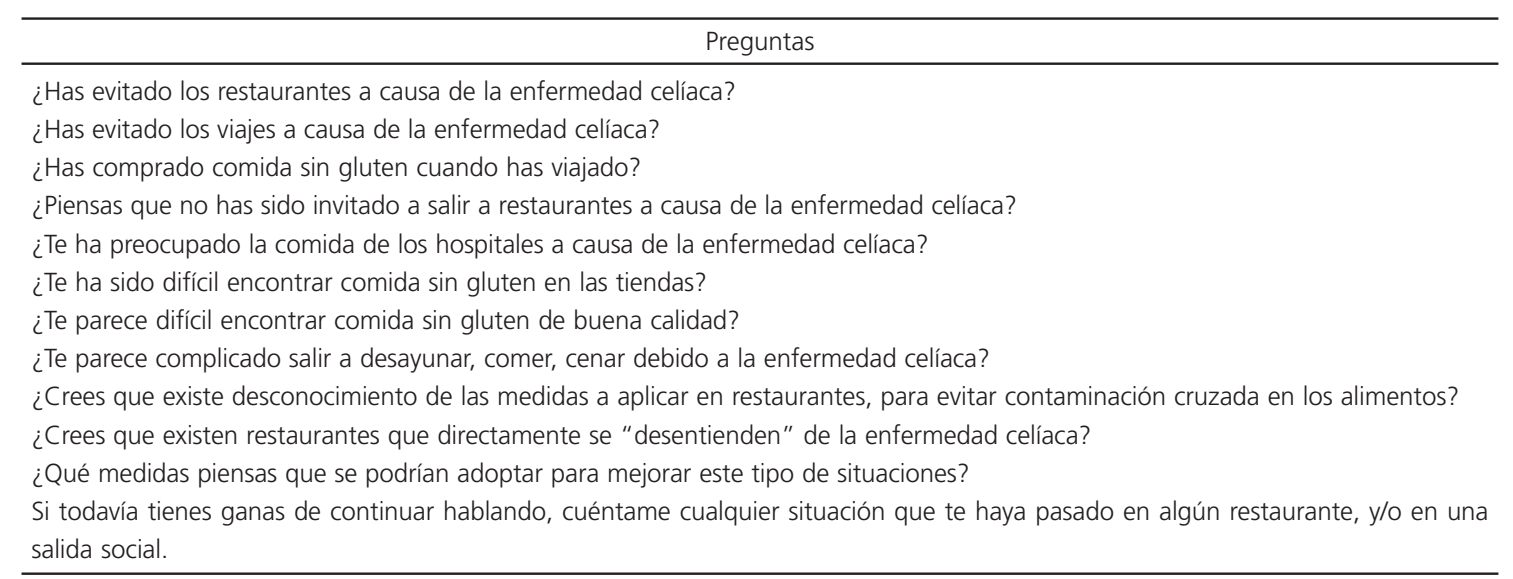

Tres investigadores realizaron el análisis de los datos desde la perspectiva de la Teoría Fundamentada con el objetivo de conocer las percepciones sobre su enfermedad de las personas celíacas, para generar una explicación teórica. La recogida, análisis e interpretación de datos se realizó simultáneamente en un proceso circular a través del método de comparación constante ${ }^{19}$, que implicó movimientos de avance y retroceso entre las transcripciones, memos (registros escritos sobre el análisis o con pautas para los investigadores) y la revisión de la literatura ${ }^{19}$.

Durante el análisis se hallaron los conceptos específicos que explicaron las percepciones sociales que tiene la enfermedad celíaca mediante los procesos de codificación abierta, axial y selectiva, y el método de comparación constante ${ }^{19}$. En la codificación abierta se etiquetaron los conceptos emergentes organizándolos en categorías. Durante la codificación axial las categorías fueron jerárquicamente relacionadas con sus subcategorías, comenzando a reagrupar los datos fracturados en la codificación abierta. Finalmente, la codificación selectiva integró las categorías, organizándolas alrededor de un concepto explicativo central, para construir la teoría sustantiva ${ }^{19}$.

Todos los participantes lo hicieron de manera voluntaria e informada. Además, debe hacerse notar que en todo momento se ha guardado el anonimato de la persona y la confidencialidad de la información a lo largo de todo el estudio y en futuras presentaciones públicas de resultados, garantizándose de esta manera cualquier problema ético futuro que pudiera surgir en relación a la investigación, siguiendo los principios de la declaración de Helsinki. Los datos recolectados a través de las entrevistas, fueron grabados en ficheros de audio con contraseña y a los cuales solo los autores tienen acceso $^{23}$.

\section{RES U LT A D OS}

En la Figura 1 se muestra el mapa conceptual resultante de las categorías teóricas emergentes que explican los problemas de adaptación del paciente celíaco tras el análisis de la información aportada por los/las celíacos del estudio. En él quedan representadas gráficamente las diferentes categorías y subcategorías vinculadas a la categoría central "Educación Sanitaria", así como la relación que se establece entre ellas, formando las bases de la teoría sustantiva en las Teorías Emergentes: los adultos se sienten aislados debido a la necesidad de comer alimentos diferentes; el desconocimiento social de la celiaquía es una barrera a la hora de realizar una dieta sin gluten; la falta de información a 


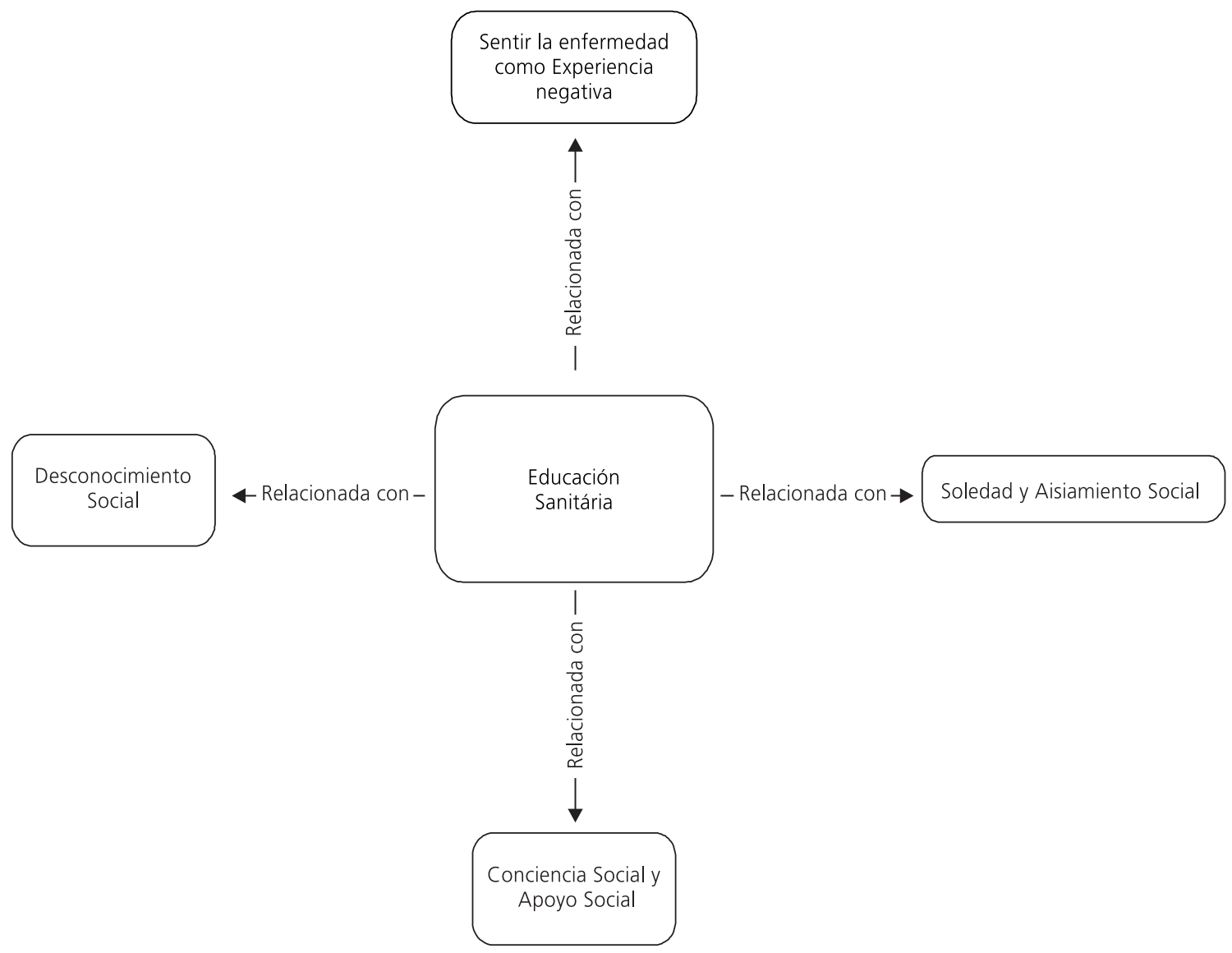

Figura 1. Categorías teóricas emergentes.

todos los niveles, restaurantes, viajes, hoteles, causa preocupación en las personas celíacas. Por último, en el Cuadro 2 podemos ver el resumen de las citas textuales recogidas que muestra la visión de las personas con enfermedad celíaca. Los resultados se organizaron en una categoría principal que es la educación sanitaria, y a través de la cual se relacionan las categorías secundarias que son: conciencia social y apoyo social, sentir la enfermedad como experiencia negativa, soledad y aislamiento social, desconocimiento social. Verbatims extraídos de las entrevistas. España (Enero-Abril, 2013)

\section{Sentir la enfermedad como experiencia negativa}

Los participantes del estudio sienten la enfermedad como una experiencia negativa continua. Manifiestan que esta experiencia es producto, de todas las situaciones desagradables que les han ocurrido a lo largo de su enfermedad ${ }^{1-3}$ (Cuadro 2).

\section{Mayor conciencia social y mayor apoyo social}

Se evidencia la falta de una mayor conciencia social, acompañada de un mayor apoyo social a todos los niveles. Esta, enfermedad pasa muy desapercibida y solo la conoce la gente más próxima al paciente celíaco ${ }^{4-6}$ (Cuadro 2).

Todas las personas celíacas demandan un aumento de información acerca de lo que significa productos no contaminados de gluten. Muchos de ellos salen a la calle con comida en sus bolsos y mochilas por miedo a esa contaminación, no 
encontrar productos disponibles, o encontrarlos a precios mucho mayores que el resto de productos $^{4-6}$ (Cuadro 2).

\section{Soledad y aislamiento social}

\section{Cuadro 2, una celíaca nos contó que} "Algunos viajes me frenan por miedo a no encontrar alimentos adaptados: países de Oriente, viajes totalmente concertados, tours por varias ciudades, Italia por el tema de la pasta, entre otros, me lleva a no realizarlos" (E03M). Este comentario va en relación con la entrevistada que nos cuenta "Sí que me han invitado alguna vez pero soy yo misma la que rechaza la invitación" (E02M). Y todos van en consonancia con "Por desgracia, no saben lo difícil que es, los que cada día luchamos por los efectos nocivos de la intolerancia al gluten" (E17H).

Cuadro 2. Verbatims extraídos de las entrevistas. España (Enero-Abril, 2013).

\section{Verbatims}

Sentir la enfermedad como experiencia negativa.

1. "Antes de pasar por eso siempre el mismo menú, comida "de enfermo" pollo deshuesado con puré de patata. ¡Si ya a 2 meses de tener esta enfermedad tengo que pasar por esto no me quiero imaginar unos años más!" (E01M)

2. "Pedí una ración de jamón ibérico sin pan, pues ya le expliqué anteriormente que no podía tomarlo y automáticamente me trajo el jamón encima de tostadas y al decirle que si no recordaba lo que le había dicho, él me dijo que lo apartara con la mano simplemente. No me comí la ración, indudablemente" (E03M)

3. "Ha sido un trauma para mí" (EO5M)

Mayor conciencia social y Mayor apoyo social.

4. "Debería haber una mayor conciencia social de lo que es la celiaquía ya que es una enfermedad que pasa desapercibida y que solo conoce la gente más próxima al paciente celíaco. Muchos de los conocidos de un celíaco no sabrán ni que lo es. Además se cree que es una enfermedad que afecta a una minoría de la población y últimamente esto está cambiando. Cada vez se diagnostican más casos y es más normal encontrarse con un celíaco, un producto etiquetado sin gluten o una sección en un supermercado única y exclusiva para celíacos. Creo que los restaurantes deberían subirse al carro" (E11M)

5. "En general no saben a qué me refiero cuando menciono la enfermedad, ni tampoco saben de las consecuencias que podría haber en una contaminación cruzada" (E14M)

6. "Esta enfermedad limita mucho, sobre todo en los desayunos y meriendas. Las comidas y cenas se basan básicamente en ensaladas o verduras a la plancha y en carne o pescado también a la plancha" (E03M)

Soledad y Aislamiento Social.

7. "Algunos viajes me frenan por miedo a no encontrar alimentos adaptados: países de Oriente, viajes totalmente concertados, tours por varias ciudades, Italia por el tema de la pasta, entre otros" (E03M)

8. "Sí que me han invitado alguna vez pero soy yo misma la que rechaza la invitación" (E02M)

9. "Que la camarera cada vez que me servía un plato se reía de mí y decía "sin harina". Venía de nuevo con otro plato, se reía y otra vez decía "sin harina". (Me lo tomé a cachondeo pero a mí no me gusta ser objeto de mofa)" (E05M)

Desconocimiento Social.

10. "Es una responsabilidad muy gorda.....y no solo en los restaurantes sino también en colegios, guarderías, campamentos, ludotecas... creo que la sociedad no está ni preparada ni concienciada sobre este tema...queda mucho trabajo por hacer" (E07M)

11. "El desconocimiento de la enfermedad celíaca y sus cuidados" (E16M)

12. "Desconocimiento de la enfermedad y tampoco mostrar mucho interés por el tema" (E01M)

Educación Sanitaria.

13. "Formación clara y simple; colocar algunos carteles informativos que indiquen los riesgos de contaminación y los alimentos o productos que podrían contener gluten, etc." (EO3M)

14. "Dar muchísima información desde las comunidades autónomas o ayuntamientos y que fuera obligatorio que una vez al año por lo menos tuvieran un curso de formación sobre la enfermedad celíaca" (E04M)

15. "Por un lado información. Falta mucha información respecto a la enfermedad celíaca (qué es y lo importante qué es para nosotros llevar una dieta 100\% libre de gluten). Y por otro formación. Que en los restaurantes sepan qué productos podemos tomar, cuáles no, etc." (E21M) 
La soledad pasa a ser un aspecto importante. Sentirse excluido o forzar la propia exclusión social por parte del celíaco es un hecho, que ocurre a diario, y que de alguna manera debemos salvar y evitar.

El aislamiento social es una situación frecuente en los celíacos. El celíaco siente una falta de compañía, de afecto y de apoyo que se agrava por la carencia de relaciones sociales de calidad. Entre las posibles causas se encuentran varias circunstancias a las que el celíaco debe hacer frente en su vida: la dificultad para encontrar restaurantes, y la existencia de cierto rechazo por parte de la sociedad, en especial hoteles y restaurantes. Todo esto conduce a una privación de apoyo emocional, físico y económico ante la que el celíaco tiene menor capacidad de respuesta ${ }^{7,8,9}$.

En relación con el desconocimiento social ${ }^{10-12}$, (Cuadro 2), todos lo tienen claro: debemos concienciar a la gente en guarderías, colegios, y televisión para un mejor conocimiento de la enfermedad celíaca. Respecto a la educación sanitaria, los entrevistados afirman:

"Formación clara y simple; colocar algunos carteles informativos que indiquen los riesgos de contaminación y los alimentos o productos que podrían contener gluten, etc." (E03M).

"Dar muchísima información desde las comunidades autónomas o ayuntamientos y que fuera obligatorio que una vez al año por lo menos tuvieran un curso de formación sobre la enfermedad celíaca"(E04M).

"Por un lado información. Falta mucha información respecto a la enfermedad celíaca (qué es y lo importante que es para nosotros llevar una dieta $100 \%$ libre de gluten). Y por otro formación. Que en los restaurantes sepan qué productos podemos tomar, cuáles no, etc."(E21M).

\section{DISCUSIÓN}

De los resultados del estudio emergen las siguientes teorías: 1) Los adultos se sienten aislados debido a la necesidad de comer alimentos diferentes; 2) El desconocimiento social de la celiaquía es una barrera a la hora de realizar una dieta sin gluten; 3) La falta de información a todos los niveles, restaurantes, viajes, hoteles, causa preocupación en las personas celíacas.

En consonancia con otros estudios, se encontró que los adultos a menudo se sentían aislados o desiguales a causa de su necesidad de comer alimentos diferentes ${ }^{10,24,25}$. Las líneas de investigación deben reorientarse hacia ese camino. Debemos realizar acciones que hagan sentirse a la persona normal, vivir con tranquilidad y que su enfermedad la lleven como una experiencia positiva en su quehacer diario.

Existe un problema social causado por la distribución general de alimentos sin gluten que es similar a los casos de otras alergias alimentarias, con una repercusión en los rituales familiares ${ }^{26}$. Al igual que en otros estudios, las experiencias de nuestros participantes revelaron el impacto adicional de este aislamiento en procesos de familia, incluyendo cambios en los patrones de socialización, la exclusión de celebraciones, comidas con la familia, la alteración de los destinos de vacaciones, y los cambios en la naturaleza y frecuencia de las interacciones sociales, para dar cabida a la dieta libre de gluten. Por lo tanto, la enfermedad celíaca tiene un impacto no sólo en el individuo sino también en su entramado social 27,28

Por otro lado, los cuidados en las enfermedades crónicas pueden resultar poco gratificantes porque no se ven resultados $y$, en ese sentido, pueden propiciar un progresivo aislamiento de la persona o de la familia. Como contrapartida pueden desarrollarse nuevas redes sociales desde los dispositivos de salud, de servicios sociales o de los grupos de autoayuda que pueden venir a instrumentar y también a servir de soporte emocional. La red social personal de la familia puede ser un elemento importante en la enfermedad celíaca.

El desconocimiento general de la sociedad sobre lo que significa tener celiaquía surgió como 
otra barrera importante para el cumplimiento de la dieta. Esto es consistente con otros estudios que ponen de relieve la educación sobre la enfermedad celíaca y la dieta sin gluten como componentes necesarios de la gestión de la dieta libre de gluten ${ }^{25,29}$.

Así pues, resulta importante establecer las consecuencias que puede tener la Enfermedad Celíaca en los niños y sus familias puesto que, por las diferencias que existen con otras enfermedades crónicas, se puede tender a minimizarlo, y una prueba de ello es las pocas investigaciones a este respecto ${ }^{28}$. En este sentido, una de nuestras limitaciones que puede ser resuelta en un futuro es continuar esta línea de investigación, en la cual se abordarían los problemas desde el punto de vista de los padres que tienen hijos con la enfermedad celíaca, abordando siempre la situación en dependencia a la edad y conocimiento sobre la enfermedad que pueda tener el niño.

Existen además limitaciones como pueden ser que la mayoría de los participantes tenían medios económicos suficientes para comprar y preparar los alimentos sin gluten y fueron capaces de hacerlo de manera que se ha facilitado la adherencia a la dieta. Los estudios futuros deben considerar la inclusión de una muestra más diversa y estudiar la manera de llegar a las familias que no participan en las actividades de apoyo celíacos.

\section{CONCLUSIÓN}

Como conclusión final es importante situar cuál es el coste real emocional que les supone a los pacientes celíacos o a sus familias la presencia de esta enfermedad crónica; al mismo tiempo, la investigación de esta cuestión podrá redundar en afinar en el tipo de intervenciones más adecuadas para ellos, planteando la necesidad de aplicar nuevas formas de intervención sociosanitarias que complementen las actuales, y que permitan dar una respuesta global a las necesidades de salud del paciente afectado por la enfermedad celíaca.

\section{COLABORADORES}

JR ALMAGRO contribuyó en la concepción y el diseño de la investigación, la recogida de datos, el análisis y la interpretación de los datos, la redacción del artículo y aspectos teóricos. MCS RUIZ, JS GONZÁLEZ, G BACIGALUPE y AH MARTíNEZ contribuyeron en la redacción del artículo, en la revisión crítica y en la aprobación de la versión final del artículo.

\section{REFERÊ NCIAS}

1. Rostom A, Dube C, Cranney A, Saloojee N, Sy R, Garritty C, et al. Celiac disease. Evid Rep Technol Assess (Summ). 2004; 104:1-6.

2. Mustalahti K, Catassi C, Reunanen A, Fabiani E, Heier M, McMillan S, et al. The prevalence of celiac disease in Europe: Results of a centralized, international mass screening project. Ann Med. 2010; 42(8):587-95.

3. World Gastroenterology Organisation. Enfermedad Celiaca. Wisconsin (EEUU): WGO; 2005.

4. Rewers M. Epidemiology of celiac disease: What are the prevalence, incidence, and progression of celiac disease? Gastroenterol. 2005; 128(4 Suppl. 1):S47-51.

5. Catassi C, Kryszak D, Bhatti B, Sturgeon C, Helzlsouer K, Clipp SL, et al. Natural history of celiac disease autoimmunity in a USA cohort followed since 1974. Ann Med. 2010; 42(7):530-8.

6. Lohi S, Mustalahti K, Kaukinen K, Laurila K, Collin $\mathrm{P}$, Rissanen $\mathrm{H}$, et al. Increasing prevalence of coeliac disease over time. Aliment Pharmacol Ther. 2007; 26(9):1217-25.

7. Lionetti $E$, Catassi $C$. New clues in celiac disease epidemiology, pathogenesis, clinical manifestations, and treatment. Int Rev Immunol. 2011; 30(4):219-31.

8. Tack GJ, Verbeek WHM, Schreurs MWJ, Mulder CJJ. The spectrum of celiac disease: Epidemiology, clinical aspects and treatment. Nat Rev Gastroenterol Hepatol. 2010; 7(4):204-13.

9. Dewar DH, Ciclitira PJ. Clinical features and diagnosis of celiac disease. Gastroenterology. 2005; 128(4 Suppl. 1):S19-24.

10. Zarkadas M, Dubois S, Maclsaac K, Cantin I, Rashid $M$, Roberts KC, et al. Living with coeliac disease and a gluten-free diet: A Canadian perspective. J Hum Nutr Diet. 2013; 26(1):10-23.

11. Barratt SM, Leeds JS, Sanders DS. Quality of life in coeliac disease is determined by perceived degree of difficulty adhering to a gluten-free diet, not the 
level of dietary adherence ultimately achieved. J Gastrointest Liver Dis. 2011; 20(3):241-5.

12. Fabiani E, Taccari LM, Ratsch IM, Di Giuseppe S, Coppa G V, Catassi C. Compliance with glutenfree diet in adolescents with screening-detected celiac disease: A 5-year follow-up study. J Pediatr. 2000; 136(6):841-3.

13. Rosland A-M, Heisler M, Piette JD. The impact of family behaviors and communication patterns on chronic illness outcomes: A systematic review. J Behav Med. 2012; 35(2):221-39.

14. Santos S, Crespo C, Silva N, Canavarro MC. Quality of life and adjustment in youths with asthma: The contributions of family rituals and the family environment. Fam Process. 2012; 51(4):557-69.

15. Fiese BH, Hammons A, Grigsby-Toussaint D. Family mealtimes: A contextual approach to understanding childhood obesity. Econ Hum Biol. 2012; 10(4):365-74.

16. Flick U. Designing qualitative research. London: Sage; 2008.

17. Charmaz K. "Discovering" chronic illness: Using grounded theory. Soc Sci Med. 1990; 30(11):1161-72.

18. De la Cuesta-Benjumea C. La Teoría fundamentada como herramienta de análisis. Rev Cult los Cuid. 2006; 10(20):136-40.

19. Strauss A, Corbin J. Basic of qualitative research. Techniques and procedures for developing Grounded Theory. London: Sage; 1998.

20. Jeon Y-H. The application of grounded theory and symbolic interactionism. Scand J Caring Sci. 2004; 18(3):249-56.

21. Strauss A, Corbin J. Bases de la investigación cualitativa. Técnicas y procedimientos para desarrollar la teoria fundamentada. $2^{\text {a }}$ ed. Colombia: Universidad de Antioquia; 2002.
22. Taylor S, Bogdan R. Introducción a los métodos cualitativos de investigación. Barcelona: Paidós; 1998.

23. Bryant A, Charmaz K. The sage handbook of grounded Theory. London: Sage; 2007 [cited 2013 Feb 2]. Available from: http://srmo.sagepub.com/ view/the-sage-handbook-of-grounded-theory/ SAGE.xml

24. Bongiovanni TRS, Clark AL, Garnett EA, Wojcicki $J M$, Heyman MB. Impact of gluten-free camp on quality of life of children and adolescents with celiac disease. Pediatrics. 2010; 125(3):e525-9. http://dx. doi.org/10.1542/peds.2009-1862

25. Byström I-M, Hollén E, Fälth-Magnusson K, Johansson $A$. health-related quality of life in children and adolescents with celiac disease: From the perspectives of children and parents. Gastroenterol Res Pract. 2012; 2012:1-6.

26. Cummings AJ, Knibb RC, King RM, Lucas JS. The psychosocial impact of food allergy and food hypersensitivity in children, adolescents and their families: A review. Allergy. 2010; 65(8):933-45.

27. Rouf $K$, White L, Evans K. A qualitative investigation into the maternal experience of having a young child with severe food allergy. Clin Child Psychol Psychiatry. 2012; 17(1):49-64.

28. Bacigalupe G, Plocha A. Celiac is a social disease: Family challenges and strategies. Fam Syst Health. 2015; 33(1):46-54.

29. Sverker A, Ostlund G, Hallert C, Hensing G. Sharing life with a gluten-intolerant person--the perspective of close relatives. J Hum Nutr Diet. 2007; 20(5):412-22.

Recibido: enero 15, 2016 Versión final: mayo 3, 2016 Aprobado: julio 1, 2016 